\title{
The rational zero point and reinforcement
}

\author{
WILLIAM L. CROLL \\ Carleton University, Ottawa 1, Canada
}

Haaf (1971) proposed a scaling procedure for partitioning rewards from punishments for studies of human learning. The present paper argues that: (a) this scaling procedure has not been adequately validated; (b) it can never be successfully validated because it ignores the relative nature of reinforcement; and (c) when it is extended to account for the relativity of reinforcement, it becomes unnecessarily complex.

Haaf (1971) noted that it has become increasingly common in studies of human (especially child) learning to define incentive value in terms of paired-comparison judgments. Thus, a child might be asked whether he prefers receiving a piece of candy or a marble and then be placed in a learning situation where obtaining one of these incentives is contingent upon performing some instrumental response. Presumably, performance in the learning task would be better if the child's preferred incentive were used. Haaf next argued that, although the paired-comparison procedure represents an improvement over earlier methods of defining incentive value, there is an important limitation inherent in the paired-comparison procedure. This limitation is that, although the procedure yields relative incentive values, it does not yield absolute incentive values. For example, if candy were rated as preferred over a marble, we would know that candy has a higher incentive value than does a marble, but we would not know whether: (a) candy is a larger reward than is a marble, (b) candy is a reward and a marble is a punishment, or (c) candy is a less severe punishment than is a marble. The reason for this uncertainty, Haaf suggested, is that the paired-comparison procedure does not yield a "rational zero point," such that events with positive values function as rewards and events with negative values function as punishments. Then Haaf described a scaling procedure, based upon earlier work by Jones (1967), which presumably does yield such a rational zero point. This procedure, in addition to requiring comparisons between pairs of single events, requires comparisons of single events with multiple events. Thus, the child would indicate his preference for candy vs a marble but would also indicate his preference for candy vs the combined package of candy and a marble. The data obtained from this package procedure are then used to calculate a zero scale point, which is then used to partition rewards from punishments, i.e., events with positive values should function as rewards and events with negative values should function as punishments. Imbedded in this procedure is the assumption that the obtained zero point will partition rewards from punishments no matter what the instrumental response happens to be in the subsequent learning task.
Although the package scaling procedure has proved useful in the scaling of preferences (Bock \& Jones, 1968), in the present paper it will be argued that the zero scale point yielded by this scaling procedure does not provide a satisfactory partition for separating rewards from punishments. It will be argued that: (a) the procedure has not yet been validated adequately with regard to its intended purpose; (b) in its present form, the procedure can never be validated successfully because it ignores the relative nature of reinforcement; and (c) when the procedure is extended to account for the relativity of reinforcement, it becomes unnecessarily complex.

Consider first the problem of validation. The package scaling procedure is valid only if the zero scale point does in fact partition rewards from punishments. There is, however, nothing in the scaling procedure itself which guarantees that this will occur. It is an empirical question whether events with positive and negative values will function as rewards and punishments, and the only way in which validation can be attempted is to use events with positive and negative values in a learning experiment and see whether they do function as assumed. This same point has been made in a similar context by Gormezano (1966). Such an experiment has apparently not been conducted; thus, the validity of the procedure has not yet been ascertained.

Next, consider whether the package procedure would prove valid were such an experiment conducted. There is good reason to believe it would not. Premack (1971) has demonstrated that the incentive value of an event is not an absolute property of that event but that it is a relational property between the event and the instrumental response upon which it is made contingent. If the incentive is more preferred than is making the instrumental response, then the incentive will function as a reward for that response. If the incentive is less preferred than is making the instrumental response, then the incentive will function as a punishment for that response. Thus, whether a given event will function as a reward or as a punisbment cannot be specified, except with respect to a given response. Furthermore, the events which serve as rewards and punishments for one response will not in general be the same as those which serve as rewards and punishments for some other response. These facts entail that any attempt to scale the reward and punishment values of a set of events must necessarily include in the scaling procedure that response upon which the scaled events are to be made contingent in a subsequent learning situation. In other words, the scaling procedure must determine how much the $S$ prefers making the instrumental response as well as how much he prefers receiving the various incentives. The package procedure in its present form fails to do this and, therefore, cannot be successfully validated. 
In order to avoid possible confusion, an additional point needs to be made with respect to the relativity of reinforcement. There is some evidence (Jones, 1967) that the zero point yielded by the package scaling procedure remains invariant over partial changes in the set of events being scaled for preference. Although this suggests that the rational zero point may be absolute rather than relative under some conditions, it does not follow that reinforcement is also absolute rather than relative. As discussed earlier, the rational zero point provides no information about the partitioning of rewards from punishments. Thus, the question of whether the zero point is absolute or relative is completely orthogonal to the question of whether reinforcement is absolute or relative.

Finally, suppose that the package scaling procedure were extended so that the instrumental response was included in the scaling procedure. Would the package procedure then be better than a simple paired-comparison procedure that also included the instrumental response? Both procedures will partition rewards from punishments for a given instrumental response: Those events which are more preferred than the response will serve as rewards and those events which are less preferred than the response will serve as punishments. Furthermore, the partition that is obtained for a given response will not be the same as that obtained for some other response, regardless of which scaling procedure is used. Thus, there appears to be no basis for preferring the package procedure over the simple paired-comparison procedure. On the contrary, economic considerations would seem to clearly favor the simple paired-comparison procedure.

\section{REFERENCES}

Bock, R. D., \& Jones, L. V. The measurement and prediction of judgment and choice. San Francisco: Holden-Day, 1968. P. 273.

Gormezano, I. Classical conditioning. In J. B. Sidowski (Ed.), Experimental methods and instrumentation in psychology. New York: McGraw-Hill, 1966. P. 385.

Haaf, R. A. The rational zero point on incentive-object preference scales: A developmental study. Developmental Psychology, 1971, 5, 537.

Jories, L. V. Invariance of zero-point scaling over changes in stimulus context. Psychological Bulletin, 1967, 67, 153-164. Premack, D. Catching up with common sense or two ides of a generalization: Reinforcement and punishment. In R. Glaser (Ed.), The nature of reinforcement. New York: Academic Press, 1971. Pp. 121-150.

(Received for publication February 18, 1973.) 